\title{
Modelling of Dynamic Behaviour of Elastic-Plastic Structures under Impact
}

\author{
K.Q. Wu and T.X Yu \\ Department of Mechanical Engineering, Hong Kong University of Science and Technology, \\ Clear Water Bay, Kowloon, Hong Kong
}

Keywords: Modelling, Dynamic Behaviour, Elastic-Plastic Structures, Impact

\begin{abstract}
It is much more difficult to predict the dynamic behaviour of elastic-plastic structures compared with the corresponding quasi-static one because of complicated loading conditions and the effect of inertia. By assuming that the quasi-static structural and interface behaviour of a structure is specified and its dynamic deformation mode is similar to the quasi-static one, two mechanical models, namely the Stick Model and the Non-Stick Model, are proposed in order to predict the dynamic behaviour of the structure under a rigid-body impact. Each model contains two lumped masses and two linear/non-linear springs. Whilst spring 2 represents the mechanical property of the interface between the structure and the impinger, spring $\mathbf{0}$ represents the elastic-plastic behaviour of the structure, no matter the latter is strain-hardening, perfectly plastic or strain-softening in the plastic stage. With the complicated deformation history involving loading, unloading and reversed loading being taken into account, the large dynamic deformation process can be completely traced and the final deformation can be predicted by these models. Finally, to verify the validity of the mechanical models proposed for real structures, impact tests on simply supported beams were conducted and the agreement is good.
\end{abstract}

\section{INTRODUCTION}

Most engineering systems are constructed largely of simple structure members, e.g., beams, plates and shells, so that an understanding of their response is an essential prerequisite for revealing the dynamic behaviour of more complex systems. In this paper, we will consider the dynamic response of a structural member when subjected to a known impact.

Now there are three distinct directions for these investigations: establishment of empirical formulas, development of relatively simple models, and full numerical simulations. The first approach relies on extensive testing, and if good correlations can be found, they may be extremely useful for interpolation to predict other cases that differ only in minor ways from the given data set. However, they cannot be expected to give more than rudimentary insight into the basic physics of the problem. Also, because numerical simulations usually require: sophisticated computer facilities and considerable expense, considerable interest has been shown in developing the intermediate, i.e. mechanical modelling approach. The construction of mechanical models relies on approaches similar to those used successfully in strength of materials and hydraulics. Here simplifying assumptions about kinematics and internal forces are made, and the mechanics principles for mass, momentum, and energy are satisfied in integral form.

For some types of structures, such as beams, their dynamic deformation modes are usually similar to corresponding static ones. Suppose the static behaviour of an elastic-plastic structure is known, the principal objective of the present study is to predict its dynamic response when subjected to a 
known impact by means of simple mass-spring mechanical models. A simply-supported beam is taken as a typical structural member to illustrate and to experimentally verify the proposed models.

\section{STRUCTURAL IMPACT MODELS}

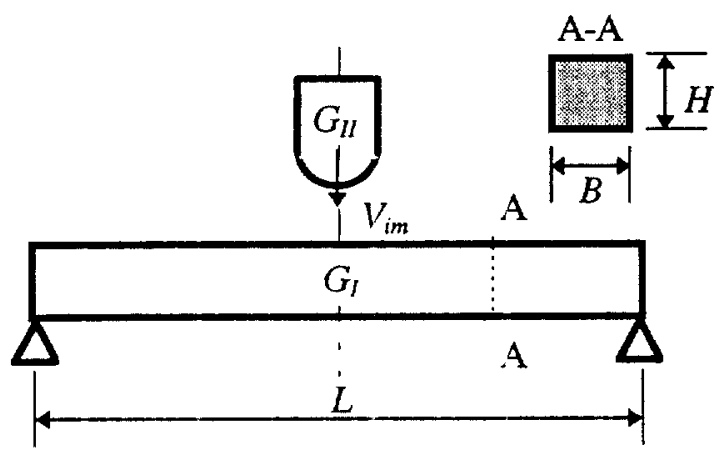

(a)

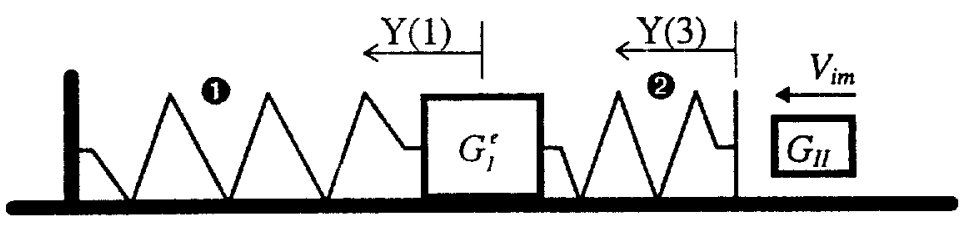

(b)

Fig. 1. (a) Impact of a simplely supported beam by a mass $G_{I I}$ travelling with a velocity $V_{i m}$. (b) Schematic illustration for mechanical models .

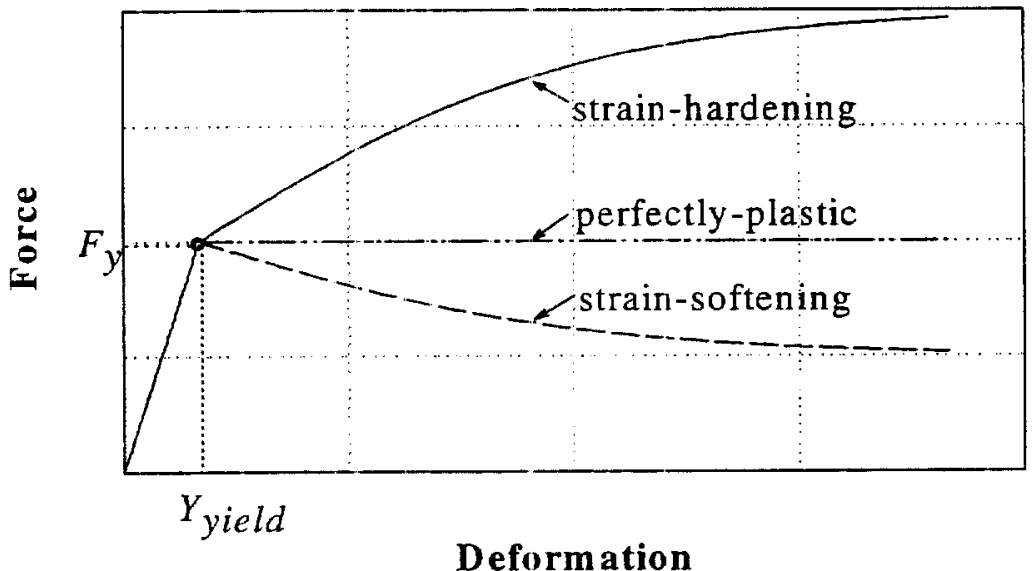

Fig. 2. Constitutive relation of spring $\mathbf{0}$

We consider a simply supported uniform beam of dimensions $L \cdot B \cdot H$ and mass $G_{I}$, as shown in Fig. 1(a), being struck at the mid-span by a rigid mass $G_{I I}$ travelling with an initial velocity $V_{i m}$.

In order to predict dynamic response of the beam under impact, two mechanical models, i.e. the Stick Model (SM) and the Non-Stick Model (NSM), are proposed. Fig. 1(b) is a schematic illustration of these two models.

These basic models are used to represent an impact between a rigid projectile and a deformable structure, such as a beam. A weightless linearly elastic spring 2 with stiffness $k_{2}$ is used to simulate the local deformability at the interface of the target structure. The static elastic-plastic structural characteristic of the target system is modeled by a weightless spring $\mathbf{0}$, which could be isotropic strain-hardening or perfectly-plastic or even strain-softening in the plastic stage. The inertia of the beam is represented by an equivalent mass $G_{I}^{e}$, which is determined by the fundmental natural 
frequency $\omega_{l}$ and structural elastic stiffness $k_{i}$ of the beam. The permanent deformation of the models is presumably the mean amplitude of the elastic vibration after plastic deformation ceases.

The constitutive relation of the elastic-plastic spring $\mathbf{0}$ is shown in Fig. 2. The relation between the resistant force and deformation is described as

$$
\mathrm{F}[Y]=\left\{\begin{array}{ll}
k_{1} \cdot Y & \text { if } Y \leq F_{y} / k_{1} ; \\
F_{y} \cdot\left[1+\eta \cdot \tanh \left(\frac{Y-F_{y} / k_{1}}{\mathrm{~K} \cdot F_{y} / k_{1}}\right)\right] & \text { if } Y>F_{y} / k_{1} .
\end{array}\right\}
$$

where $\quad F_{y}$ : yield force of spring $\mathbf{0}$.

$k_{1}$ : stiffness of spring $\mathbf{0}$ in its elastic range .

$Y$ : shortening of spring $\mathbf{0}$.

$\kappa$ : material factor, a parameter to simulate structural characteristic of the target system. Normally it is positive and depends on the material chosen.

$\eta$ : plastic factor, a parameter to represent plastic characteristic of spring $\mathbf{0}$.

$$
\begin{aligned}
& 0<\eta \leq \kappa, \text { strain-hardening; } \\
& \eta=0, \text { perfectly-plastic; } \\
& -1<\eta<0, \text { strain-softening. }
\end{aligned}
$$

The difference between the two models is the assumption on the local mechanical behavior of the surface under impact. The Stick Model is based on the assumption that impact between a rigid projectile and a target system is completely inelastic, which means that the projectile will stick on the target system throughout the entire dynamic response period after impact. As for the Non-Stick Model it is assumed that spring 2 can sustain compression only, which implies that the projectile will depart the target system if the relative displacement becomes negative during the dynamic response of the system and a multi-impact is allowed.

\section{FORMULATION}

The $Y$-coordinates shown in Fig. 1(b) are adopted, with the origins located respectively at the centers of the target $G_{I}^{e}$ and the projectile $G_{I I}$ when the latter first touches the surface of the target. The whole model is considered as a lumped mass-spring system, in which the masses of springs $\mathbf{0}$ and 2 are neglected. The target system is initially stationary; only the projectile moves with an initial speed $V_{i m}$ before impact occurs.

As impact occurs, we designate $Y(l)$ and $Y(3)$ as the two $Y$-coordinates shown in Fig. 1, to represent displacements of the target and the projectile, respectively. The differential equations of motion are obtained as:

$$
\left\{\begin{array}{l}
\ddot{Y}(1)=\Lambda \cdot k_{2} \cdot \frac{[Y(3)-Y(1)]}{G_{I}^{e}}-\frac{F[Y(1)]}{G_{I}^{e}} \\
\ddot{Y}(3)=\Lambda \cdot k_{2} \cdot \frac{[Y(1)-Y(3)]}{G_{I I}}
\end{array}\right.
$$

where for the Stick Model, $\Lambda=1$;

for the Non-Stick Model, $\Lambda=1$ if $[Y(3)-Y(I)] \geq 0$, or $\Lambda=0$ if $[Y(3)-Y(1)]<0$.

Let $\quad L_{e}=F_{y} / k_{1}, y(1)=Y(1) / L_{e}, y(3)=Y(3) / L_{e}, \tau=t \cdot \sqrt{k_{1} / G_{I}^{e}}$,

$\mathrm{F}[y(1)]=\mathrm{F}[Y(1)] / F_{y}, R_{m}=G_{I I} / G_{I}^{e}, R_{s}=k_{2} / k_{1}$. 
The following discussion will be made by employing non-dimensional variables. By using $y(2)$ and $y(4)$ to denote the non-dimensional velocities of the target and the projectile respectively, a system of first-order ordinary differential equations is obtained as

$$
\left\{\begin{array}{l}
\dot{y}(I)=y(2) \\
\dot{y}(2)=\Lambda \cdot[y(3)-y(I)] \cdot R_{s}-\mathrm{F}[y(I)] \\
\dot{y}(3)=y(4) \\
\dot{y}(4)=\Lambda \cdot[y(I)-y(3)] \cdot R_{s} / R_{m}
\end{array}\right.
$$

with the initial conditions:

$y(l)=y(3)=0, y(2)=0, y(3)=v_{i m}=\left(V_{i m} / L_{e}\right) \cdot \sqrt{G_{l}^{e} / k_{l}} \neq 0$.

Equations (3) are solved by using a fourth-order Runge-Kutta procedure with the initial conditions given in (4).

\section{EXPERIMENTAL SET-UP AND METHODS}

The experimental set-up and methods are listed in Table 1 . The punches were considered as rigid because of their considerable hardness compared with the aluminum specimens. The load or impact velocity was applied to the center and normal to the planes of the specimens. The impact velocity ranged from 2.2 to $6.3 \mathrm{~m} / \mathrm{s}$ and was obtained by adjusting the height of the drop weight or pressure of compressed air if pneumatic assistance was used. The drop weight was set as $3.528 \mathrm{~kg}, 6.288 \mathrm{~kg}$ or $13.83 \mathrm{~kg}$. All experimental data were recorded automatically by the data acquisition system attached to the Dynatup Impact Tester and the MTS.

Table 1.

\begin{tabular}{|c|c|c|c|}
\hline & \multicolumn{2}{|c|}{ Quasi-static property tests } & Dynamic property tests \\
\hline & Interface & Structural & Structural \\
\hline $\begin{array}{l}\text { Experimental } \\
\text { facility }\end{array}$ & \multicolumn{2}{|c|}{810 Material Test System } & $\begin{array}{l}\text { GRC } 8250 \text { Dynatup Drop } \\
\text { Weight Impact Tester }\end{array}$ \\
\hline Punch & \multicolumn{3}{|c|}{ with a high carbon steel spherical head $\phi 25.4 \mathrm{~mm}$ and its hardness is over HRC-40 } \\
\hline $\begin{array}{l}\text { Load or } \\
\text { impact speed }\end{array}$ & $50 \mathrm{~N} / \mathrm{s}$ & $5 \mathrm{~mm} / \mathrm{min}$ & $2.2 \sim 6.3 \mathrm{~m} / \mathrm{s}$ \\
\hline $\begin{array}{l}\text { Material of } \\
\text { specimens }\end{array}$ & \multicolumn{3}{|c|}{ Al6061-T6, strain-rate insensitive } \\
\hline $\begin{array}{l}\text { Dimensions } \\
\text { of specimens }\end{array}$ & $60 \cdot 60 \cdot 18(\mathrm{~mm})^{3}$ & $100 \cdot 10 \cdot 10(\mathrm{~mm})^{3}$ & $100 \cdot 10 \cdot 10(\mathrm{~mm})^{3}$ \\
\hline $\begin{array}{l}\text { Supporting } \\
\text { condition of } \\
\text { specimens }\end{array}$ & $\begin{array}{l}\text { placed on the platform of } \\
\text { the test machine }\end{array}$ & \multicolumn{2}{|c|}{ simply supported on two blocks which are $80 \mathrm{~mm}$ apart } \\
\hline
\end{tabular}

\section{DISCUSSION \& COMPARISON ON NUMERICAL \& EXPERIMENTAL RESULTS}

The structural and interface characteristics of the specimens are shown in Figs. 3 and 4, respectively. The relevant parameters in our mechanical models can then be determined as
$k_{2}$
$k_{I}$
$F_{y}$
$\kappa$
45.0
$\eta$
$G_{I}^{e} *$
$3167 \mathrm{~N} / \mathrm{mm}$
$3800 \mathrm{~N}$
$+0.8$
$6.46 \mathrm{~g}$

$52700 \mathrm{~N} / \mathrm{mm}$

Also, an energy ratio is defined as $R_{e}=\left(G_{I I} \cdot V_{i m}^{2} / 2\right) /\left(k_{J} \cdot L_{e}{ }^{2} / 2\right)=R_{m} \cdot v_{i m}{ }^{2}$. In the experiments this energy ratio ranged from 7 to 55 , so large plastic deformation was expected. The experimental and numerical results are shown in Table 2 and Fig. 5 . 


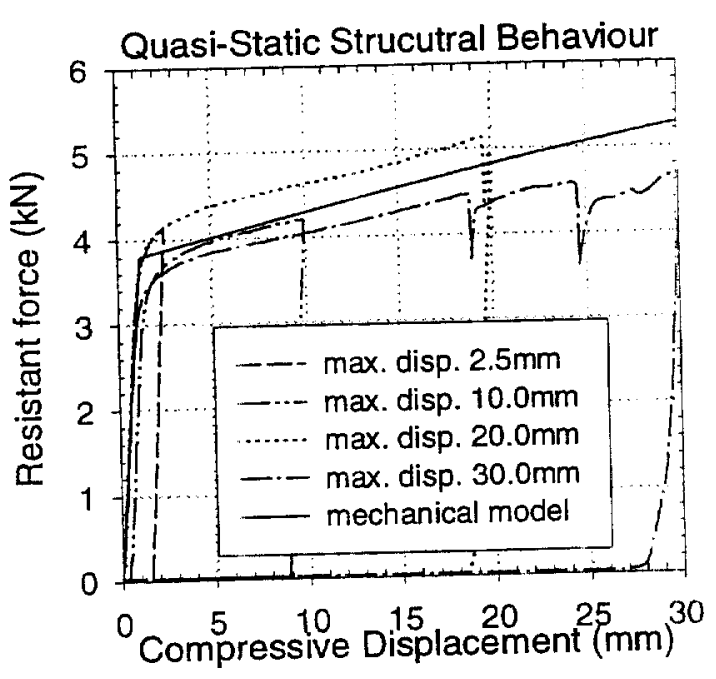

Fig. 3.

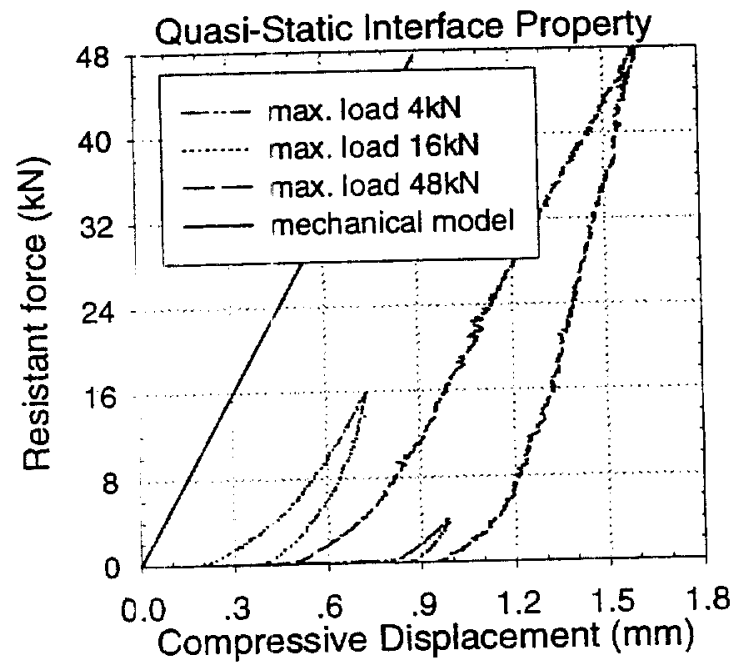

Fig. 4.

Although there exist some discrepancies between theoretical predictions and experiments, the accuracy of the numerical prediction is acceptable. It is noted that when the Stick Model (SM) is used the prediction is closer to the experimental results, which implies under an impact with large mass ratio the dynamic response process is more like the mechanism displayed by the Stick Model.

An important observation is that both the models tend to over-predict the dynamic deformation, especially when the energy ratio is very large. It may be attributed to two factors : (i) It is evident from Fig. 4 that the interface property is non-linearly elastic-plastic. In the experiments a portion of the impact energy was dissipated by the local plastic deformation at the interface. Such a portion becomes more and more important when the impact energy increases or the projectile mass becomes very large. (ii) In the experiments the specimen was not perfectly simply-supported. The friction between the specimen and the supporters also dissipated a part of impact energy.

Table 2.

\begin{tabular}{|c|c|c|c|c|c|c|c|c|c|c|c|c|c|c|}
\hline$G_{I I}(\mathrm{~kg})$ & \multicolumn{9}{|c|}{3.528} & \multicolumn{1}{|c|}{6.288} & \multicolumn{3}{|c|}{13.083} \\
\hline$V_{i m}(\mathrm{~m} / \mathrm{s})$ & 3.73 & 3.76 & 4.20 & 4.80 & 6.04 & 2.31 & 3.23 & 4.53 & 5.48 & 6.30 & 2.24 & 2.27 & 3.16 \\
\hline$R_{e}$ & 10.76 & 10.97 & 13.65 & 17.83 & 28.23 & 7.36 & 14.39 & 28.30 & 41.41 & 54.73 & 14.39 & 14.79 & 28.65 \\
\hline $\begin{array}{c}Y(1)_{\text {exp }}^{f} \\
(\mathrm{~mm})\end{array}$ & 5.46 & 5.26 & 6.42 & 8.28 & 12.19 & 3.00 & 6.00 & 12.00 & 17.53 & 23.36 & 7.07 & 7.48 & 12.00 \\
\hline
\end{tabular}

\section{CONCLUSION}

In order to predict the dynamic response of elastic-plastic structures, two simple mechanical models, i.e. the Non-Stick Model and the Stick Model, are proposed and verified experimentally. Comparison indicates that the errors between the theoretical predictions and experimental results are acceptable, so the mechanical models are reasonable and successful.

However, if the projectile mass is light while its impact velocity is high, the local effect at the interface becomes more important, and the plastic behaviour at the interface cannot be ignored. Also, for some material which are strain-rate sensitive, the dynamic interface behaviour could greatly differ from the static counterpart. These problems will be studied further.

* $G_{I}^{e}$, the equivalent mass of the target, is determined by equating the fundamental natural frequency $\omega_{l}$ of the target system to that of the beams in the experiments. For a simply supported uniform beam $\omega_{l}=\pi^{2} \cdot \sqrt{(E \cdot I) /\left(\rho \cdot A \cdot l^{4}\right)}$;

For the target system $\omega_{l}=\sqrt{k_{I} / G_{l}^{e}}$. 

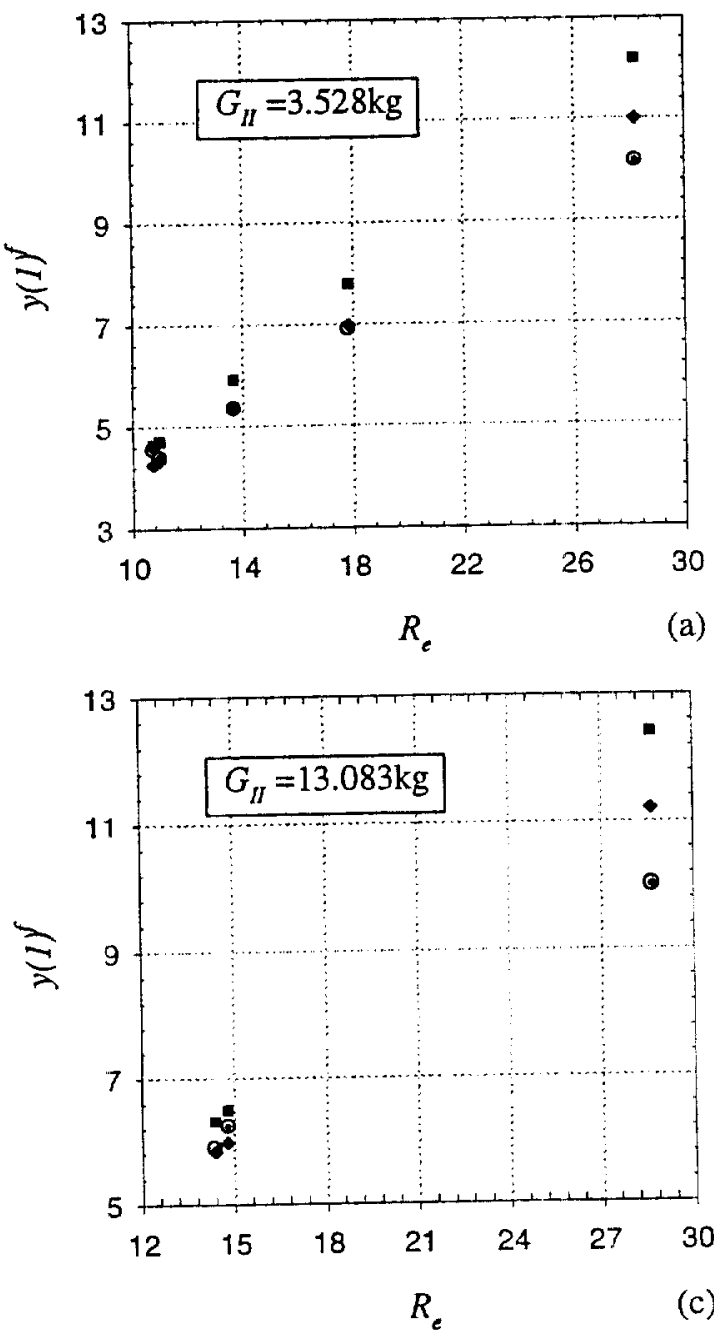

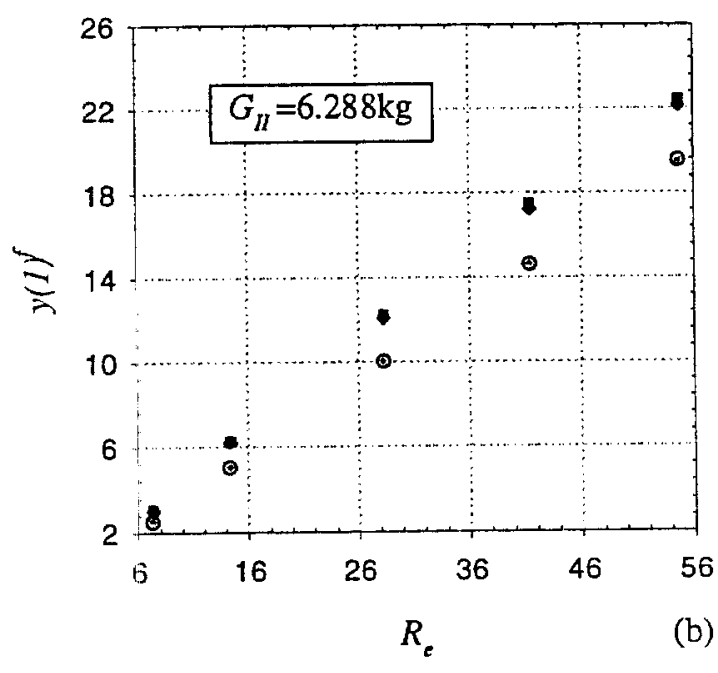

$$
\begin{array}{ll}
\text { - } & \text { experimental result } \\
\text { - } & \text { prediction by NSM } \\
\text { - } & \text { prediction by SM }
\end{array}
$$

Fig. 5 (a) (b) \& (c). Variation of the non-dimensional final deformation $y(1)^{f}$ with the energy ratio $R_{e}$.

\section{REFERENCES}

1. W.J. Stronge and T.X. Yu (1995), Dynamic Models for Structural Plasticity, Springer.

2. N. Jones (1989), Structural Impact, Cambridge University Press.

3. R.J. Harker (1983), Generalized Methods of Vibration Analysis, John Wiley \& Sons,

4. X.Y. Su, T.X. Yu and S.R. Reid (1995), Inertia-sensitive impact energy-absorbing structures, Part I: Effects of inertia and elasticity. Int. J. Impact Engng. 16, 651-672.

5. X.Y. Su, T.X. Yu and S.R. Reid (1995), Inertia-sensitive impact energy-absorbing structures, Part II: Effects of strain rate. Int. J. Impact Engng. 16, 673-689.

6. Y.P. Zhao, T.X. Yu and J. Fang (1995), On the some advances in studies of structural dynamic plastic failure, $J$. Advances in Mechanics (in Chinese) 25, 549-561.

7. L.L. Tam and C.R. Calladine (1991), Inertia and strain-rate effects in a simple plate-structure under impact loading, Int. J. Impact Engng. 11, 349-377.

8. T.G. Zhang and T.X. Yu (1989), A note on a 'velocity sensitive' energy-absorbing structure, Int. J. Impact Engng. 8, 43-51.

9. R. H. Grzebieta and N.W. Murray (1986), The static behaviour of structs with initial kinks at their centre point, Int. J. Impact Engng. 4, 147-159.

10. P.S. Symonds (1985), A review of elementary approximation techniques for plastic deformation of pulse loaded structures, Metal forming and impact mechanics, (Edited by S.R. Reid), 175195, Pergamon Press. 